\title{
Lifting the fog from the north
}

\section{A purist approach to wavefunctions can resolve some of quantum theory's infamous murkiness.}

\section{Maximilian Schlosshauer}

As physicist Richard Feynman famously observed: "Nobody understands quantum mechanics." Whereas classical physics mirrors our everyday experiences - we can comprehend the idea of a tennis ball's velocity and apply Newton's laws of motion to explain it - this is not true of quantum physics. Classical concepts, such as position and momentum, fail to consistently describe phenomena of the atomic world.

In the early twentieth century, experiments showed that microscopic entities such as electrons sometimes behave more like waves than particles. Fired through slits, they form patterns similar to overlapping ripples in a pond. This prompted quantum mechanics' founders to introduce an abstract mathematical entity - the wavefunction - to describe physical systems.

Erwin Schrödinger in the 1920s thought that the wavefunction represented real entities. He suggested that when the wavefunction is spread out in space we see wave behaviour, and when it is constricted to a narrow 'wave packet' we get a particle. $\mathrm{He}$ hit a roadblock: quantum laws implied that a wave packet describing an isolated microscopic particle would rapidly disperse, yet real particles are obviously more stable.

The 'wavefunction only' picture was dismissed for other reasons. Evolving smoothly, the wavefunction seemed unable to account for processes that appear discontinuous in space and time. Quantum mechanics also predicted that wavefunctions would take strange shapes corresponding to a superposition of many classical-looking structures, such as Schrödinger's cat, doomed while hidden in its box to be in a state of being both alive and dead.

Physicists then reinterpreted the wavefunction as a symbolic device specifying the probabilities of measurement outcomes. This produced a dualism between the wavefunction - assumed to encapsulate everything about a system's physical state - and the random measurement outcomes, the quantum events. How do these events arise? And why can they be expressed in terms of classical variables?

Niels Bohr, one of the minds behind the Copenhagen interpretation of quantum mechanics, refused to go further. Thus descended the infamous 'fog from the north'. Classical measuring apparatuses took centre stage, precipitating the wavefunction's 'collapse' to give the experimental outcome. A dichotomy between quantum and classical was introduced. Incompatible classical

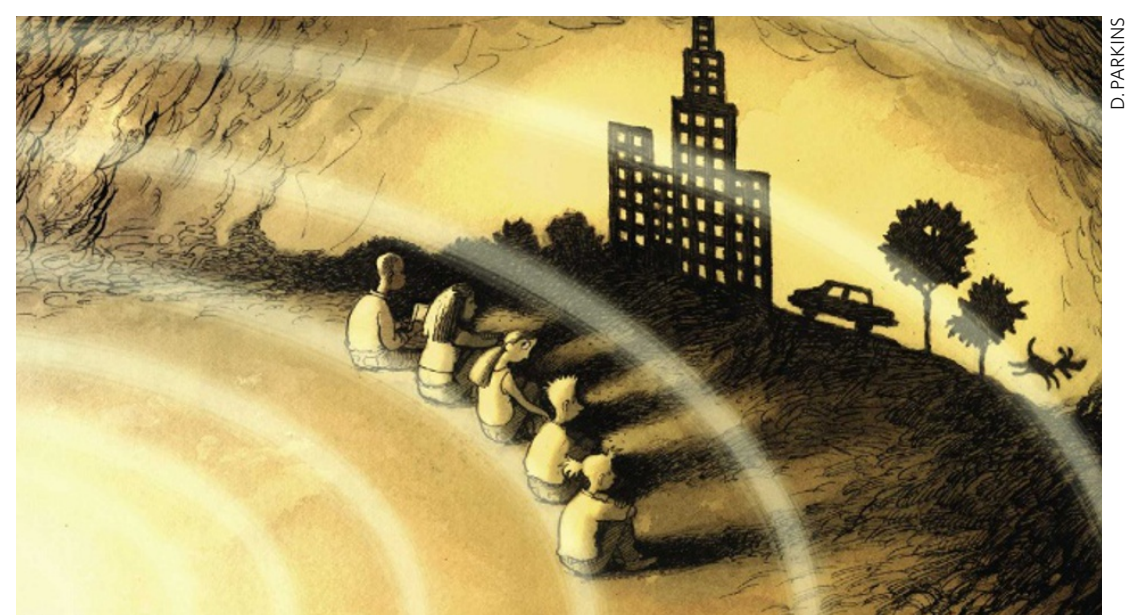

The classical world of our experience is a projected shadow in a quantum universe.

descriptions were sanctified using terminology such as wave-particle duality and complementarity (the doctrine that such descriptions must be associated with mutually exclusive experimental arrangements). Shadows of Copenhagen also hang over recent suggestions that quantum mechanics is fundamentally about information.

To lift the fog we should see the wavefunction as more than a tool for calculating probabilities. The wavefunction alone may suffice to understand both micro- and macroscopic phenomena once quantum mechanics is applied consistently.

A key ingredient is 'entanglement': when systems interact they lose their individuality and must be described by a shared wavefunction. Entanglement is ubiquitous. Physical systems cannot avoid interacting with their environment, so a system's behaviour is dictated by the wavefunction involving both system and environment. This is the physical process of quantum decoherence (see page 22). Decoherence censors the system so that only classical-looking structures are usually observable, such as objects in well-defined places. Observations, too, can be described as entangling interactions, without resorting to dualism.

Rapid yet continuous decoherence processes also explain quantum events. When an electron hits a photographic plate, it is not a mysterious act of 'measurement' that causes the electron's wavefunction to act as a point particle, but the entanglement between electron and plate (and its environment). The resulting decoherence establishes 'robustly narrow' wave packets that appear as particle-like entities, close to Schrödinger's idea.

The entangled wavefunction still involves a superposition of classical structures, such as locations of spots on a photographic plate. Because we cannot fully control the environment (such as air molecules scattering off this page), such a superposition is usually hidden from our view. Still, why is a single spot here and not there? All such observations can be thought of as temporally extended and - thanks to decoherence - effectively autonomous 'branches' of the wavefunction. Arguably, this is all we need. Introducing some novel collapse process that rids the wavefunction of unobserved branches may offer some comfort but will risk disproof by experiment, although new physics may always be discovered.

Experimentalists have created many counterintuitive 'Schrödinger kittens' including large molecules in a superposition of two distinct locations, and superpositions of electric current running in opposite directions. These have shown how decoherence leads to the continuous emergence of classical properties. Such observations can be explained using the 'pure quantum' picture of entangled wavefunctions, supporting the view that all classical structures are apparent. There is no need to introduce classical apparatuses, particles, quantum events, complementarity or wave-particle duality as fundamental concepts.

Taking the wavefunction seriously points to a quantum universe that is rather different from the everyday world. But as Feynman cautioned us, what may look like a paradox is often only a conflict between reality and your feeling of what reality ought to be. Maximilian Schlosshauer is a research fellow at the School of Physics, University of Melbourne, Melbourne, Victoria 3010, Australia. He is author of Decoherence and the Quantum-to-Classical Transition.

See also pages 22 and 50. 